\title{
Non-Markovian evolution of a two-level system interacting with a fluctuating classical field via dipole interaction
}

\author{
Samaneh Hesabi ${ }^{a}$, Davood Afshar ${ }^{\mathrm{a}, \mathrm{b}, *}$, Matteo G. A. Paris ${ }^{\mathrm{c}, *}$ \\ ${ }^{a}$ Department of Physics, Faculty of Science, Shahid Chamran University of Ahvaz, Ahvaz, Iran \\ ${ }^{b}$ Center for research on Laser and Plasma, Shahid Chamran University of Ahvaz, Ahvaz, Iran \\ ${ }^{c}$ Quantum Technology Lab, Dipartimento di Fisica Aldo Pontremoli \\ Università degli Studi di Milano, I-20133 Milano, Italy
}

\begin{abstract}
We address memory effects in the dynamics of a two-level open quantum system interacting with a classical fluctuating field via dipole interaction. In particular, we study the backflow of information for a field with a Lorentzian spectrum, and reveal the existence of two working regimes, where memory effects are governed either by the energy gap of the two-level system, or by the interaction energy. Our results shows that non-Markovianity increases with time, at variance with the results obtained for dephasing and despite the dissipative nature of the interaction, thus suggesting that the corresponding memory effects might be observed in practical scenarios.
\end{abstract}

Keywords: non-Markovianity, memory effects, stochastic electromagnetic field

\section{Introduction}

The dynamics of a closed quantum system is reversible and the time evolution of its states, which is governed by the Schrödinger equation, is described by unitary maps. On the other hand, for an open quantum system interacting with its environment, the state evolution is no longer reversible and is described by completely-positive, tracepreserving (CPTP) maps, which themselves result from the partial trace of the (unitary) joint evolution of the open system + environment. In turn, the evolution equation for an open quantum system, the so-called Master equation (ME), should be derived from the Schrödinger equation of the overall system, upon tracing out the degree of freedom of the environment [1, 2].

In most cases, obtaining a ME is quite challenging and, in turn, there are only few examples of open quantum systems for which an exact ME may be derived. In the other cases, the most common approximations used to derive the ME of an open quantum systems are those referred to as the Born and Markov approximations [1]. The Born approximation amounts to assume a weak coupling between the open system and its

\footnotetext{
*Corresponding authors

Email addresses: da_afshar@yahoo.com (Davood Afshar), matteo.paris@fisica.unimi.it (Matteo G. A. Paris) 
environment. Markov approximation consists instead in assuming that the dynamics of the environment is slow compared to the system's one. In the Markovian regime, the state of the system at time $t$ is independent on its past, i.e. we do not need to go backwards in time to account for memory effects. In turn, the loss of memory effects corresponds to CPTP maps of the Lindblad type [4, satisfying the so-called divisibility property.

Lindblad-Markov MEs are valid tools in describing the dynamics of several systems, but they are unable to describe coherent phenomena occurring in several solid-state and biological systems [5, 6, 7, as well as in material systems with a photonic band gap [8, 9, If memory effects cannot be neglected, the dynamics is referred to as non-Markovian. Non-Markovianity may also cause a backflow of information from the environment to the system, and this may be exploited e.g. to enhance security of quantum key distribution [10] or outperform metrological strategies based on uncorrelated states [11]. We also remind that there are MEs suitable to describe the dynamics of non-Markovian open quantum systems as for example the Nakajima-Zwanzig ME [12, 13] or the timeconvolutionless one [14, 15].

In order to characterize and quantify non-Markovianity, several figures of merit have been introduced. A measure has been proposed (BLP) on the basis of memory effect [16 and another one (RHP) in terms of entanglement of the system and its environment [17. In addition, other measures have been proposed, based on quantum Fisher information [18, mutual information [19] and temporal steering [20. In this paper, since we are interested in discussing the sources of memory effects for an atom interacting with its electromagnetic environment, we stick with the original BLP definition in terms of backflow of information.

A two-level atom interacting with its electromagnetic environment corresponds to an open quantum system [21, 22, 23, 24, 25, 26, 27. A well-known and solvable model to describe its dynamics is the Jaynes-Cummings one, describing the interaction with a single-mode in terms of the dipole moment [28, 29, 30]. In recent years, some studies have been performed about the non-Markovianity of this system in the presence of various environments. In particular, non-Markovianity has been considered for a two-level system coupled to a single mode of the field via the Jaynes-Cummings model using BLP measure [28, and coupled to random external fields using different measures [31]. The non-Markovianity of a damped Jaynes-Cummings model [32, 33] and for the coupling to a quantized bosonic field [21] has been studied as well.

If a two-level system is exposed to a fluctuating field with a broad spectrum, the equations of motion are not linear and the response of the system may not obtained easily. In those situations, it is convenient to employ semiclassical stochastic methods [34, 35, 36, 37, 38, to address the time evolution. Since this is a common situation of practical interest for various applications, we here investigate the non-Markovianity of a two-level system interacting with a stochastic field with a Lorentzian spectrum.

The paper is structured as follows. In Section 2 we describe our model, solve the equations of motion for the specific case of a Lorenzian spectrum and introduce the BLP measure of non-Markovianity. Our results and the different working regimes are then illustrated in Section 3. Section 4 closes the paper with some concluding remarks. 


\section{The system and the interaction model}

We consider a two-level system interacting with a classical, possibly fluctuating, e.m. field. The dynamics of the system is described by the Hamiltonian

$$
H=H_{0}-\boldsymbol{\mu} \cdot \boldsymbol{E},
$$

where $H_{0}=\frac{1}{2} \Omega \sigma_{3}$ is the free Hamiltonian of the two-level system, $\boldsymbol{\mu}=\mu \cos \alpha \sigma_{1}$ is the dipole moment of the system, and $\boldsymbol{E} \equiv \boldsymbol{E}_{t}$ is an external, possibly fluctuating, electromagnetic field, $\alpha$ is the angle between the dipole moment and the field. The field may be a deterministic function of time, describing a driving field, or a stochastic processing, describing a fluctuating background field. The latter situation is that of interest in this work. The state of the system at the time $t$ is given by

$$
\rho_{t}=\mathbb{E}\left[\left|\psi_{t}\right\rangle\left\langle\psi_{t}\right|\right]_{E},
$$

where $\mathbb{E}[\cdots]_{E}$ denotes the average over the different realizations of the background field, intended as a stochastic process. The single-realization state of the system is given by $\left|\psi_{t}\right\rangle=a_{t}|0\rangle+b_{t}|1\rangle$, where $|j\rangle, j=0,1$ are the eigenstates of the free Hamiltonian, $H_{0}|j\rangle=E_{j}|j\rangle$, with $\left|a_{t}\right|^{2}+\left|b_{t}\right|^{2}=1, \forall t$. The instantaneous dipole moment and energy of the system are given by

$$
\begin{aligned}
& M_{t}=\mu\left(a_{t} b_{t}^{*}+a_{t}^{*} b_{t}\right), \\
& W_{t}=\frac{1}{2} \Omega\left(\left|b_{t}\right|^{2}-\left|a_{t}\right|^{2}\right),
\end{aligned}
$$

where we already employed natural units, i.e. $\hbar=1$. In turn, the Schrödinger equation for the two-level systems may be written in terms of $M_{t}$ and $W_{t}$ as follows

$$
\begin{aligned}
\ddot{M}_{t}+\Omega^{2} M_{t} & =-(2 \mu \cos \alpha)^{2} W_{t} E_{t}, \\
\dot{W}_{t}+\beta_{s}\left(W_{t}+\frac{1}{2} \Omega\right) & =\dot{M}_{t} E_{t},
\end{aligned}
$$

where $\beta_{s}$ is the Einstein coefficient for spontaneous emission. The solution of the above equations is given by [34, 35]:

$$
\begin{aligned}
M_{t}= & M_{0} \cos \Omega t+\dot{M}_{0} \frac{\sin \Omega t}{\Omega}-\frac{(2 \mu \cos \alpha)^{2}}{\omega} \int_{0}^{t} d s \sin [\Omega(t-s)] W_{s} E_{s}, \\
W_{t}= & -\frac{1}{2} \Omega+\left(W_{0}+\frac{1}{2} \Omega\right) e^{-\beta_{s} t}+\int_{0}^{t} d s W_{s} G_{t s} \\
& -M_{0} \int_{0}^{t} d s \sin \Omega s e^{-\beta_{s}(t-s)} E_{s}+\dot{M}_{0} \int_{0}^{t} d s \cos \Omega s e^{-\beta_{s}(t-s)} E_{s},
\end{aligned}
$$

where

$$
G_{t s}=-(2 \mu \cos \alpha)^{2} e^{-\beta_{s}(t-s)} \int_{s}^{t} d y E_{y} E_{s} \cos \Omega(s-y)
$$


and $M_{0}, W_{0}$ denotes the initial values of the dipole moment and the energy, respectively. In the following, we assume that the field amplitude fluctuates around a vanishing average $\mathbb{E}\left[E_{t}\right]_{E}=0$. In this case, we have

$$
\begin{aligned}
\mathbb{E}\left[M_{t}\right]_{E} & =M_{0} \cos \Omega t \\
\mathbb{E}\left[W_{t}\right]_{E} & =\frac{1}{2 \pi i} \oint d z \frac{e^{z t}\left[z W_{0}-\frac{1}{2} \beta_{s} \Omega\right]}{z\left[z+\beta_{s}+(2 \mu \cos \alpha)^{2} F(z)\right]} \\
F(z) & =\frac{z}{2} \int_{-\infty}^{\infty} d \omega \frac{I(\omega)}{(\omega-\Omega)^{2}+z^{2}},
\end{aligned}
$$

where $I(\omega)$ is the power spectrum of field, i.e. the Fourier transform of its correlations function $\mathbb{E}[E(t) E(s)]_{E}$. On the assumption that the background radiation field has a Lorentzian spectrum centered at the resonance $\Omega$, i.e.

$$
I(\omega)=\frac{I_{0} \beta^{2}}{\left[(\omega-\Omega)^{2}+\beta^{2}\right]},
$$

$\mathbb{E}\left[W_{t}\right]_{E}$ may be simplified to

$$
\mathbb{E}\left[W_{t}\right]_{E}=A\left[-1+e^{-\gamma t} \cos \lambda t\right]+W_{0} e^{-\gamma t} \cos \lambda t+\frac{1}{2} e^{-\gamma t} \beta_{s} \frac{\sin \lambda t}{\lambda}(B-A),
$$

where

$$
\begin{array}{rlrl}
A & =\frac{\Omega \beta_{s}}{2 \beta_{s}+\pi I_{0}(2 \mu \cos \alpha)^{2}}, & B=\frac{\Omega\left[\beta-\pi I_{0}(2 \mu \cos \alpha)^{2}\right]}{2 \beta_{s}+\pi, I_{0}(2 \mu \cos \alpha)^{2}}, \\
\gamma=\frac{1}{2}\left(\beta+\beta_{s}\right), & \lambda^{2}=\frac{1}{2}(2 \mu \cos \alpha)^{2} \pi \beta I_{0}-\frac{1}{4}\left(\beta-\beta_{s}\right)^{2} .
\end{array}
$$

Using Bloch representation in terms of the Pauli matrices, the evolved state of the system, i.e. $\rho_{t}$ in Eq. 22, may thus rewritten as

$$
\rho_{t}=\frac{1}{2}\left(\mathbb{I}+\mathbb{E}\left[W_{t}\right]_{E} \sigma_{3}+\mathbb{E}\left[M_{t}\right]_{E} \sigma_{1}\right)
$$

which says that the dynamics of coherence does depend only on its energy gap $\Omega$, whereas the populations are affected also by the variables governing the interaction Hamiltonians. The purity of the state at time $t$ is given by

$$
\mu_{t}=\operatorname{Tr}\left[\rho_{t}^{2}\right]=\frac{1}{2}\left(1+\mathbb{E}\left[M_{t}\right]_{E}^{2}+\mathbb{E}\left[W_{t}\right]_{E}^{2}\right) .
$$

\subsection{Quantification of memory effects}

Before addressing the dynamics of non-Markovianity in details, let us briefly review how memory effects (due to non-Markovian dynamics) may be quantified using the time dependence of the trace distance $D\left(\rho_{1 t}, \rho_{2 t}\right)=\frac{1}{2}\left\|\rho_{1 t}-\rho_{2 t}\right\|$ between a pair of evolved states of the system [16]. In the previous formula, $\|A\|$ denotes the trace-norm of the operator $A$ i.e. $\|A\|=\operatorname{Tr}[|A|]=\operatorname{Tr}\left[\sqrt{A^{\dagger} A}\right]=\sum_{k} \sqrt{\left|a_{k}\right|}, a_{k}$ being the eigenvalues of $A^{\dagger} A$. 
As a starting point, we remind that any completely-positive and trace-preserving map $\mathcal{E}_{t}$ is also contractive, i.e. the trace distance between any two evolved states decreases $D\left(\rho_{1 t}, \rho_{2 t}\right)<D\left(\rho_{10}, \rho_{20}\right)$, where $\rho_{t}=\mathcal{E}_{t}\left[\rho_{0}\right]$. For any Markovian process, the divisibility property makes the contractivity properties to hold for any two chosen values of time, i.e.

$$
D\left(\rho_{1 t}, \rho_{2 t}\right)<D\left(\rho_{1 s}, \rho_{2 s}\right) \quad \forall t>s .
$$

In turn, this monotonic decrease of distinguishability may be understood as an irreversible flow of information from the system to the environment. On the other hand, for a nonMarkovian process, divisibility is lost, and the trace distance may increase in some timeinterval. This means that in those cases information is flowing back from the environment to the system. This property may be employed to define a measure of non-Markovianity as follows:

$$
N_{T}=\max _{\rho_{10}, \rho_{20}} \int_{\sigma>0}^{T} d s \sigma\left(s, \rho_{1 s}, \rho_{2 s}\right),
$$

where $\sigma\left(t, \rho_{1 t}, \rho_{2 t}\right)$ is the rate of change of the trace distance

$$
\sigma\left(t, \rho_{1 t}, \rho_{2 t}\right)=\frac{d}{d t} D\left(\rho_{1 t}, \rho_{2 t}\right)
$$

and the maximisation is performed over all the possible pairs of initial states. The integration is meant over the intervals where $\sigma$ is positive up to a maximum time $T$, which corresponds to a maximum interaction time, e.g. due to a finite observation time or, in case of a propagating system, due to the finite size of the region with nonzero field.

\section{Dynamics of non-Markovianity}

Using the expression in Eq. 16, the trace distance between a generic pair of evolved states may be written as follows

$$
D\left(\rho_{1 t}, \rho_{2 t}\right)=\frac{1}{2} \sqrt{e^{-\gamma t}\left(W_{10}-W_{20}\right)^{2} \cos ^{2} \lambda t+\left(M_{10}-M_{20}\right)^{2} \cos ^{2} \Omega t} .
$$

The non-Markovianity measure $N_{T}$ corresponds to the maximal possible backflow of information, and is calculated by taking the maximum over all the initial pairs of states using Eq. 191. For qubit systems the maximum is obtained for a pair of states that are pure and orthogonal [16, 39] and this means that we may write

$$
\left(W_{10}-W_{20}\right)^{2}=4 \cos ^{2} \theta \quad\left(M_{10}-M_{20}\right)^{2}=4 \sin ^{2} \theta \quad \theta \in[0, \pi / 2] .
$$

The maximisation over pairs of states is thus transformed into a maximisation over the single state parameter $\theta$, and the trace distance rate may be written as

$$
\begin{aligned}
\sigma\left(t, \rho_{1 t}, \rho_{2 t}\right) & \equiv \sigma(t, \gamma, \lambda, \Omega, \theta) \\
& =-\frac{1}{2 S}\left[e^{\frac{1}{2} \gamma t} \cos ^{2} \theta \Omega \sin 2 \Omega t+e^{-\frac{1}{2} \gamma t} \cos ^{2} \theta\left(\gamma \sin ^{2} \lambda t+\lambda \sin 2 \lambda t\right)\right] \\
S & =\sqrt{e^{\gamma t} \cos ^{2} \theta \cos ^{2} \Omega t+\sin ^{2} \theta \cos ^{2} \lambda t} .
\end{aligned}
$$


Before going to the explicit analysis of non-Markovianity, it is useful to notice a scaling property of $\sigma$, i.e.

$$
\sigma(t, \gamma, \lambda, \Omega, \theta)=\gamma \sigma(\gamma t, 1, \lambda / \gamma, \Omega / \gamma, \theta),
$$

and, in turn, of $N_{T}$,

$$
N_{T}(\gamma, \lambda, \Omega)=N_{\gamma T}(1, \lambda, \Omega) .
$$

As a consequence, one is led to consider the following dimensionless variables

$$
t \rightarrow \tau=\gamma t, \quad \Omega \rightarrow \Omega / \gamma, \quad \lambda \rightarrow \lambda / \gamma, \quad T \rightarrow \gamma T,
$$

which amounts to measure all the considered quantities in unit of $\gamma^{-1}$. With this choice one has

$$
N_{T} \equiv N_{T}(\lambda, \Omega)=\max _{\theta} \int_{\sigma>0}^{T} d \tau \sigma(\tau, 1, \lambda, \Omega, \theta) .
$$

Numerical analysis shows that for any given $T$, the maximum is obtained either for $\theta=0$ or for $\theta=\pi / 2$, depending on the values of $\lambda$ and $\Omega$. Upon exploiting this result, we may write

$$
N_{T}(\lambda, \Omega)=\int_{0}^{T} d \tau \max \left[g_{0}(\tau, \Omega), g_{\pi / 2}(\tau, \lambda)\right]
$$

where

$$
\begin{aligned}
g_{0}(\tau, \Omega) & =\frac{1}{2}[\sigma(\tau, 1, \lambda, \Omega, 0)+|\sigma(\tau, 1, \lambda, \Omega, 0)|] \\
& =\frac{\Omega}{4} \frac{|\sin 2 \Omega \tau|-\sin 2 \Omega \tau}{|\cos \Omega \tau|} \\
g_{\pi / 2}(\tau, \lambda) & =[\sigma(\tau, 1, \lambda, \Omega, \pi / 2)+|\sigma(\tau, 1, \lambda, \Omega, \pi / 2)|] \\
& =\frac{1}{8} e^{-\tau / 2} \frac{2\left|\cos ^{2} \lambda \tau+\lambda \sin 2 \lambda \tau\right|-1-\cos 2 \lambda \tau-2 \lambda \sin 2 \lambda \tau}{|\cos \Omega \tau|} .
\end{aligned}
$$

As a first consistency check, let us consider short time evolution, which should correspond to a Markovian behaviour. Indeed, we have

$$
\begin{aligned}
\sigma(\tau, 1, \lambda, \Omega, 0) & \simeq-\Omega^{2} \tau+O\left(\tau^{2}\right), \\
\sigma(\tau, 1, \lambda, \Omega, \pi / 2) & \simeq-\frac{1}{2}+\left(\frac{1}{4}-\lambda^{2}\right) \tau+O\left(\tau^{2}\right),
\end{aligned}
$$

such that $N_{T}=0$. Using Eq. 27) we have then evaluated non-Markovianity as a function of $\lambda$ and $\Omega$ for different values of the medium "length" $T$. Results are illustrated in Fig. 1. as it is apparent from the plots, non-Markovianity is as an increasing function of both $\lambda$ and $\Omega$. Moreover, for increasing $\Omega, N_{T}$ becomes almost independent on $\lambda$. In Fig. 1, we show both the values obtained for $\theta=0$ (light gray, referred to as the $\Omega$-region in the following) and $\theta=\pi / 2$ (dark gray, $\lambda$-region) in order to illustrate the regions in the $\lambda-\Omega$ 
plane where each parameter is more relevant; remind that for $\theta=0, N_{T}$ does depend only on $\Omega$ and vice versa for $\theta=\pi / 2$. The non-Markovianity is the maximum between the two values. Notice that non-Markovianity increases with time, despite the dissipative nature of the interaction, and this fact suggests that the corresponding memory effects may be observed in practice. For sake of completeness we report the analytic expression of $N_{T}(\Omega)$ in the $\Omega$-region, the corresponding expression in the $\lambda$-region is cumbersome and will not be reported here,

$$
N_{T}(\Omega)=[[\Omega T]]+\frac{1}{2}(|\cos \Omega T|-|\sin \Omega T| \cot \Omega T),
$$

where $[[x]]$ denotes the integer part of $x$.
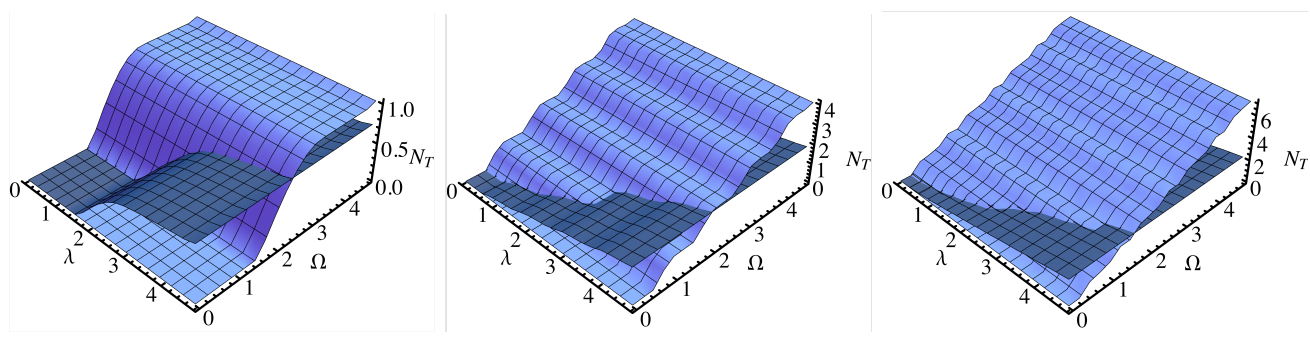

Figure 1: Non-Markovianity $N_{T}$ versus $\lambda$ and $\Omega$ and for three different fixed values of "length" of the medium $T$. From left to right $T=1,3,5$. The two surfaces denote the integral in Eq. 27] in the $\Omega$-region, i.e. for $\theta=0$ (light gray) and in the $\lambda$-region, i.e. $\theta=\pi / 2$ (dark gray). The non-Markovianity $N_{T}$ is the maximum between the two values. $N_{T}$ vanishes for small values of $\lambda$ and $\Omega$ and increases with both, as well as with the time $T$ (notice the different ranges on the $N_{T}$ axes).

In Fig. 2 we show the behaviour of $N_{T}$ as a function of $T$ for different values of $\lambda$ and $\Omega$. In the left panel we show $N_{T}$ vs $T$ for $\lambda=0.1$ and few values of $\Omega$, whereas in the central panel we show $N_{T}$ vs $T$ for $\Omega=0.1$ and few values of $\lambda$. As it is apparent from the plots, $N_{T}$ increases continuously, but not smoothly. Roughly speaking, $N_{T}$ is growing linearly with $T$ when $\Omega$ is the relevant parameter and sub-linearly viceversa. Notice the different range for $N_{T}$ in the two panels. In the right panel we summarise the results, showing the region in the $T-\lambda-\Omega$ parameter space where $N_{T}=\int_{0}^{T} d \tau g_{\pi / 2}(\tau, \lambda) \equiv N_{T}(\lambda)$, i.e. where $\lambda$ is the relevant parameter determining the non-Markovianity. As mentioned before, this physically corresponds to have a background field with a broad spectrum compared to the width of the transition. Since random telegraph noise (RTN) is characterized by a Lorentzian spectrum, our results may be compared to those obtained for a two-level system interacting with a dephasing classical environment fluctuating according to RTN [37. It turns out that dipole interaction is leading to more pronounced non-Markovian effects compared to dephasing: backflow of information increases with time instead of oscillating, and no threshold on the width of the spectrum (or the switching rate of the process) appears. This is a remarkable result, in view of the ubiquitous occurrence of the dipole interaction in nature. It may be also appropriate at this point to remind that the non-Markovian character of a quantum map resulting from the interaction with a classically fluctuating field is fully independent on the nature of the stochastic process used to describe its time dependence [40, 41]. In other words, the stochastic process describing the field may be (classically) Markovian, whereas the quantum map originating from the 
interaction with the corresponding classical field may be (quantum) non-Markovian at all times. This is exactly what happens for the telegraph noise, which is described by a classically memoryless continuous-time stochastic process, but it leads to a non-Markovian quantum process if the switching rate is small enough [37.
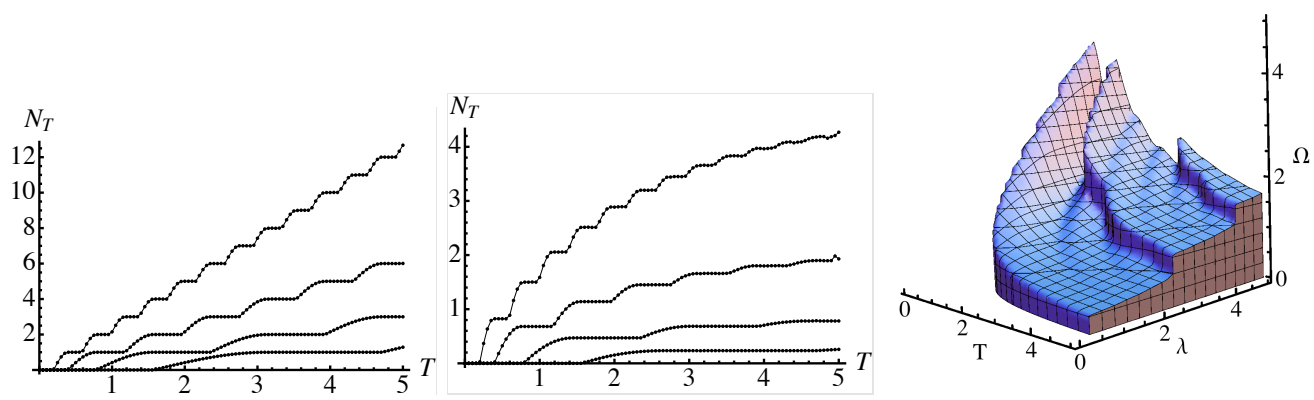

Figure 2: Non-Markovianity $N_{T}$ as a function of $T$ for different values of $\lambda$ and $\Omega$. In the left panel we show $N_{T}$ vs $T$ for $\lambda=0.1$ and (from bottom to top) $\Omega=1,2,4,8$, whereas in the central panel we show $N_{T}$ vs $T$ for $\Omega=0.1$ and (from bottom to top) $\lambda=1,2,4,8$. Notice the different range for $N_{T}$ in the two panels. The right panel shows the region in the $T-\lambda-\Omega$ parameter space where $\int_{0}^{T} d \tau g_{\pi / 2}(\tau, \lambda)>\int_{0}^{T} d \tau g_{0}(\tau, \Omega)$ (in the range $T \in[0,5], \lambda \in[0,5], \Omega \in[0,5]$ ).

\section{Conclusions}

In this paper, we have addressed the dynamics of a two-level system immersed in a classical fluctuating field, and interacting with its environment via dipole interaction. We have discussed the non-Markovianity of the corresponding quantum evolution, as measured by the backflow of information (BLP measure) and, in particular, we have evaluated the backflow of information for a background field with a Lorentzian spectrum, also comparing our result with the analogue dephasing case.

Our results uncovered the existence of two working regimes, which we referred to as $\Omega$-regime or $\lambda$-regime, corresponding to sharp and broad spectrum of the field, where memory effects are governed either by the energy gap of the two-level system, or by the interaction energy, respectively. Non-Markovianity vanishes for short interaction times, independently on $\lambda$ and $\Omega$ and then increases monotonically with time and with both $\lambda$ and $\Omega$, despite the dissipative nature of the interaction, thus suggesting that the corresponding memory effects may be observed in practical scenarios. When compared to the corresponding dephasing dynamics, it turns out that dipole interaction is leading to more pronounced non-Markovian effects, with the backflow of information that increases with the interaction time independently on width of the (Lorentzian) field spectrum.

\section{Acknowledgements}

DA was supported by Shahid Chamran University of Ahvaz [Grant No. 96/3/02/16670]. MGAP is member of GNFM-INdAM. 


\section{References}

\section{References}

[1] H-P. Breuer and F. Petruccione, The Theory of Open Quantum Systems, (Oxford University Press, Oxford, 2002).

[2] U. Weiss, Quantum dissipative systms, (World Scientific, Singapore, 2008).

[3] H. J Carmichael and M. O Scully, Phys. Today. 53, 78 (2000).

[4] G. Lindblad, Comm. Math. Phys. 48, 119, (1976).

[5] S. Maniscalco and F. Petruccione, Phys. Rev. A 73, 012111, (2006).

[6] X.-T. Liang, Phys. Rev. E 82, 051918, (2010).

[7] C. W. Lai, P. Maletinsky, A. Badolato, and A. Imamoglu, Phys. Rev. Lett. 96, 167403, (2006) .

[8] A. Chiuri, C. Greganti, L. Mazzola, M. Paternostro and P. Mataloni, Sci. Rep. 2, 968, (2012).

[9] P. Lambropoulos, G. M. Nikolopoulos, T. R. Nielsen, and S. Bay, Rep. Prog. Phys. 63, (2000) 455.

[10] R. Vasile, S. Olivares, M. G. A. Paris, S. Maniscalco Phys. Rev. A 83, 042321 (2011).

[11] A. W. Chin, S. F. Huelga, M. B. Plenio Phys. Rev. Lett. 109, 233601 (2012).

[12] S. Nakajima, Progr. Theor. Phys. 20, 968, (1958).

[13] R. Zwanzig, Physica 30, 1109 (1964).

[14] N. Hashitsume, F. Shibata, and M. Shingu, J. Stat. Phys. 17, 155 (1977); F. Shibata, Y. Takahashi, and N. Hashitsume, J. Stat. Phys. 17, 171 (1977).

[15] S. Chaturvedi and F. Shibata, Z. Phys. B 35, (1979).

[16] E.-M. Laine, J. Piilo and H.-P. Breuer, Phys. Rev. A 81, 062115, (2010).

[17] A. Rivas, S. F. Huelga, and M. B. Plenio, Phys. Rev. Lett. 105, 050403, (2010).

[18] X.-M. Lu, X. Wang and C. P. Sun, Phys. Rev. A 82, 042103, (2010).

[19] S. Luo, S. Fu and H. Song, Phys. Rev. A 86, 044101, (2012).

[20] S.-L. Chen et al., Phys. Rev. Lett. 116, 020503, (2016).

[21] C. Addis, B. Bylicka, D. Chrus̀ciǹski, and S. Maniscalco, Phys. Rev. A 90, 052103, (2014).

[22] T. J. G. Apollaro, C. Di Franco, F. Plastina and M. Paternostro, Phys. Rev. A 83, 032103, (2011).

[23] Z. Xu, W. Yang, and M. Feng, Phys. Rev. A 81, 044105, (2010).

[24] Y.-J. Zhang, W. Han, Y.-J. Xia, J.-P. Cao, and H. Fan, Sci. Rep. 4, 4890, (2014).

[25] B. Bellomo, G. Compagno, R. L. Franco, A. Ridolfo, S. Savasta, Phys. Scripta T147, 014004, (2012).

[26] C. Pineda, T. Gorin, D. Davalos, D. A. Wisniacki, and I. Garcia-Mata, Phys. Rev. A 93, 022117, (2016).

[27] Z. X. Man, N. B. An, and Y. J. Xia, Phys. Rev. A 90, 062104, (2014).

[28] A. Smirne, L. Mazzola, M. Paternostro and B. Vacchini, Phys. Rev. A 82, 062114, (2010).

[29] S. Alipour, A. Mani and A. T. Rezakhani, Phys. Rev. A 85, 052108, (2012).

[30] H.-S. Zeng, N. Tang, Y.-P. Zheng, and G.- Y. Wang, Phys. Rev A 84, 032118,(2011).

[31] M. Mannone, R. Lo Franco and G. Compagno, Phys. Scr. T153, 014047, (2013).

[32] Y. J. Zhang, Y. J. Xia, and H. Fan, EPL 116, 30001, (2016).

[33] J.-G. Li, J. Zou, and B. Shao, Phys. Rev. A 81, 062124,(2010).

[34] F. W. Cummings, Am. J. Phys. 30, 898, (1962).

[35] F. W. Cummings, Nuovo Cim. B 70, 102, (1982).

[36] F. Benatti, R. Floreanini, S. Olivares, Phys. Lett. A 376, 2951 (2012).

[37] C. Benedetti, M. G. A. Paris, S. Maniscalco, Phys. Rev. A 89, 012114 (2014).

[38] M. G. A. Paris, Physica A 413, 256 (2014).

[39] S. Wissmann, A. Karlsson, E.-M. Laine, J. Piilo, and H.-P. Breuer, Phys. Rev. A 86, 062108, (2012).

[40] B. Vacchini, A. Smirne, E.-M. Laine, J. Piilo and H.-P. Breuer New J. Phys. 13, 093004 (2011).

[41] S. Cialdi et al., Appl. Phys. Lett. 110, 081107 (2017). 